\title{
Avaliação da superfície de materiais resinosos expostos ao óxido de alumínio
}

\section{Evaluation of resinous material surfaces exposed to aluminum oxide powder}

\section{Lourdes dos SANTOS-PINTO}

Livre Docente - Professora Adjunto - Departamento de Clínica Infantil - Faculdade de Odontologia de Araraquara Universidade estadual Paulista - UNESP - Araraquara - SP - Brasil.

\section{Rita de Cássia Loiola CORDEIRO}

Livre Docente - Professora Adjunto - Departamento de Clínica Infantil - Faculdade de Odontologia de Araraquara Universidade estadual Paulista - UNESP - Araraquara - SP - Brasil.

\section{Luciana Monti LIMA}

Mestre e Doutoranda - Programa de Pós-graduação em Ciências Odontológicas, área de Odontopediatria - Faculdade de Odontologia de Araraquara - Universidade estadual Paulista - UNESP - Araraquara - SP - Brasil.

\section{Victoria A. MARKER}

Engenheira de Materiais - Texas - USA

\begin{abstract}
Resumo
O objetivo deste estudo foi avaliar o efeito da deposição de óxido de alumínio sobre restaurações resinosas adjacentes a área tratada pelo sistema de abrasão a ar. Foram utilizados 10 terceiros molares devidamente seccionados e incluídos em cera utilidade deixando exposta a face vestibular. Em cada espécime confeccionou-se com a caneta de alta rotação, 4 preparos cavitários de aproximadamente $2 \mathrm{~mm}$ de diâmetro, eqüidistantes de uma linha central demarcada na cera. As 40 cavidades foram restauradas aleatoriamente com resina composta (Silux-3M) e selante resinoso (FluoroshieldDentsply). Em seguida, réplicas de cada espécime foram obtidas pela moldagem com silicone (Imprint II-3M) e modelo em resina epóxi (Stycast 1266- Emerson \& Cummings). Na seqüência, foram feitas 2 aplicações de $15 \mathrm{~s}$ do jato abrasivo na parte central dos espécimes, utilizando-se o aparelho PrepStar ${ }^{\circledR}$ regulado a 80 psi, com ponta ativa de $45^{\circ}$ e $0,48 \mathrm{~mm}$ de diâmetro interno posicionada a $5 \mathrm{~mm}$ da superfície. Ao final de cada aplicação, as superfícies foram lavadas, secas e moldadas novamente. Todas as réplicas foram avaliadas em MEV por 2 examinadores calibrados que fizeram uma avaliação cega quanto ao tipo de restauração. Os dados tabulados em escores foram analisados estatisticamente por análise de variância de Friedman. Os resultados evidenciaram que não houve diferença estatística significativa entre as superfícies das restaurações analisadas antes e após a utilização do jato abrasivo ( $p>0,05)$. Uma vez que o jato de óxido de alumínio não foi aplicado diretamente sobre as restaurações, provavelmente a baixa energia cinética das partículas periféricas não foi capaz de produzir alterações em suas superfícies.
\end{abstract}

\section{UNITERMOS}

Abrasão Dental por Ar; desgaste de restauração dentária; óxido de alumínio 


\section{INTRODUÇÃO}

No início dos anos 50, o sistema de preparo por abrasão foi introduzido na Odontologia com o objetivo de reduzir a pressão, o calor, a vibração e o ruído presentes durante o preparo mecânico do dente com brocas. Devido à falta de precisão dos preparos cavitários, requisito básico para a utilização dos materiais restauradores existentes, e, com a introdução dos motores de alta rotação que permitiram obter cavidades com ângulos definidos, superfícies planas e paredes lisas, os sistemas abrasivos entraram em desuso ${ }^{1,18}$.

Recentemente novos aparelhos de abrasão ar foram lançados no mercado. Eles também utilizam a energia cinética gerada pelo impacto das partículas de óxido de alumínio em alta velocidade e cortam eficientemente esmalte e dentina. Esta tecnologia ressurgiu quando os modernos materiais restauradores e as técnicas adesivas tornaram possíveis as restaurações de cavidades conservadoras que não demandam precisão na forma e nem ângulos definidos ${ }^{18,20}$.

Dentre as inúmeras indicações para a utilização da abrasão a ar podemos ressaltar a limpeza e remoção de manchas, facilitando o diagnóstico da lesão de cárie em fossa e fissuras profundas ${ }^{4-6,14}$ e permitindo maior penetração dos selantes ${ }^{12}$. Esta técnica também pode ser utilizada no condicionamento mecânico do esmalte e dentina ${ }^{9,10,15}$, no preparo de cavidades ${ }^{5,6,8}$ bem como, na remoção ou no reparo de restaurações de resina composta, ionômero de vidro e selantes com infiltração ou fraturas $5,8,19$.

Goldstein \& Parkins ${ }^{10}$ (1995), avaliando os aparelhos de abrasão a ar apontaram como uma de suas desvantagens o fato das partículas de óxido de alumínio se espalharem e estas, quando entram em contato com os espelhos bucais os tornam foscos e não apropriados para o uso. Além disso, durante sua utilização as partículas abrasivas se acumulam em todo o campo operatório, o que requer do operador um aprimorado senso de direção e a utilização de um excelente sistema de sucção para que as estruturas adjacentes não sejam atingidas.

Pouco se tem estudado sobre a influência das partículas abrasivas ao atingir ou depositar-se sobre a superfície de restaurações previamente realizadas. Portanto, o objetivo deste estudo foi avaliar a superfície de restaurações em resina composta e selante resinoso expostos ao óxido de alumínio durante a utilização do sistema de abrasão a ar, tendo como hipótese nula que a deposição das partículas de óxido de alumínio não produzem alterações na superfície das restaurações existentes.

\section{Material e MÉTOdo}

Foram utilizados 10 terceiros molares obtidos de pacientes que assinaram um termo de doação e consentimento livre esclarecido aprovado pelo Comitê de Ética em Pesquisa da Faculdade de Odontologia de Araraquara (protocolo ${ }^{\circ} 75 / 04$ ).

Os dentes recém-extraídos foram mantidos em formol tamponado a $10 \%$ até o início do experimento, quando foram limpos por meio de raspagem do tecido periodontal e tiveram suas coroas seccionadas no sentido mésio-distal na máquina de corte de precisão ISOMET $^{\mathrm{TM}} 1000$ (Buehler $\left.^{\circledR}\right)$. Em seguida, todas as superfícies vestibulares dos dentes selecionados foram incluídas em blocos de cera utilidade deixando exposta apenas a superfície de esmalte.

Em todas as faces vestibulares foram realizadas, com auxílio de alta rotação e ponta diamantada esférica (1013, KG Sorensen), quatro preparos cavitários de aproximadamente $2,0 \mathrm{~mm}$ de diâmetro e profundidade, estando estes eqüidistantes de uma linha central demarcada na cera. Desta forma, 40 cavidades foram preparadas e os 10 espécimes divididos aleatoriamente em 2 grupos experimentais: Grupo 1 - cavidades restauradas com resina composta Silux - 3M; e Grupo 2 - cavidades restauradas com selante resinoso Fluoroshield $^{\circledR}$ - Dentsply. Uma resina composta e um selante foram selecionados por serem os materiais restauradores normalmente presentes em casos clínicos onde está indicado o reparo de restaurações utilizando-se como instrumento de corte o jato de óxido de alumínio.

A técnica restauradora para ambos os grupos seguiu as especificações dos fabricantes e para evitar uma possível interferência nos resultados optou-se por não realizar o acabamento e polimento das restaurações em resina composta, uma vez que estes procedimentos não seriam preconizados para o grupo do selante resinoso.

Após as restaurações, réplicas em resina epoxy (Stycast 1266, Emerson \& Cummings) foram obtidas de cada espécime por meio de moldagem com silicone (Imprint II, 3M Dental Products) e serviram como controle inicial (Réplica 1).

Na seqüência, um jato em linha reta com partículas de óxido de alumínio de $27 \mu \mathrm{m}$ foi executado na parte central do espécime, utilizando-se como guia a mesma linha central demarcada na cera utilidade para confecção das restaurações eqüidistantes (Figura 1). Utilizou-se o aparelho de abrasão a ar PrepStar ${ }^{\circledR}$ (Danville Engennering USA) regulado a 80 psi de pressão, com ponta ativa em ângulo de $45^{\circ}$ e $0,48 \mathrm{~mm}$ 


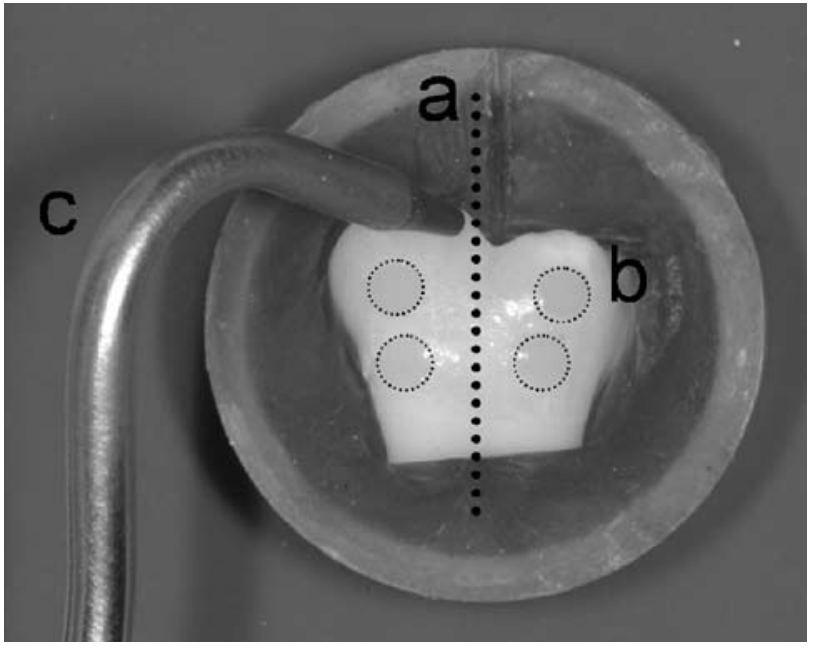

FIGURA 1: Linha central (guia) para aplicação do jato abrasivo (a); cavidades restauradas eqüidistantes da linha central (b); ponta do aparelho de abrasão a ar (c).

de diâmetro interno posicionado a $5,0 \mathrm{~mm}$ da superfície dental. Foram realizadas duas aplicações de 15 segundos do jato abrasivo, sendo que ao final de cada uma delas os espécimes foram lavados, secos e moldados para obtenção de novas réplicas em resina epoxy (Réplicas 2 e 3).

No decorrer do experimento nenhuma amostra foi perdida e todas as 30 réplicas obtidas das moldagens dos espécimes (10 inicias - controle, 10 após a primeira aplicação e 10 após a segunda aplicação do jato abrasivo), foram metalizadas, analisadas e comparadas em microscopia eletrônica de varredura (MEV) por dois examinadores previamente calibrados que atribuíram escores para cada restauração de acordo com a Tabela 1 .

Os valores obtidos foram tabulados na forma de escores e analisados estatisticamente através da análise de variância de Friedman $(p \leq 0,05)$.

\section{TABELA 1 - Critérios para atribuição de escores para a superfície do material restaurador analisado.}

\begin{tabular}{c|l}
\hline Escore & Características da superfície \\
\hline 1 & Nenhuma alteração \\
2 & Presença de ranhuras \\
3 & Presença de ranhuras e microcavidades \\
4 & $\begin{array}{l}\text { Presença de ranhuras, sulcos e } \\
\text { microcavidades }\end{array}$ \\
\hline
\end{tabular}

\section{Resultados}

Os resumos da análise de variância de Friedman aplicada para os escores atribuídos pelos dois examinadores na análise das superfícies das restaurações de resina composta e selante resinoso estão descritos, respectivamente, nas Tabela 2 e 3 , onde os resultados evidenciaram ausência de diferença estatística significativa entre as superfícies das restaurações analisadas antes e após a utilização do jato abrasivo ( $p>0,05)$.

$\mathrm{Na}$ análise das restaurações em microscopia eletrônica de varredura foi possível identificar fraturas em suas margens e alguns riscos discretos na superfície (Figura 2). Além disso, alguns espécimes apresentaram uma discreta rugosidade superficial após a segunda aplicação do jato de óxido de alumínio.

\section{Dıscussão}

O sistema de abrasão a ar vem sendo amplamente empregado no reparo marginal de restaurações realizadas com material resinoso, uma vez que permite uma remoção conservadora da estrutura dentária, limpando

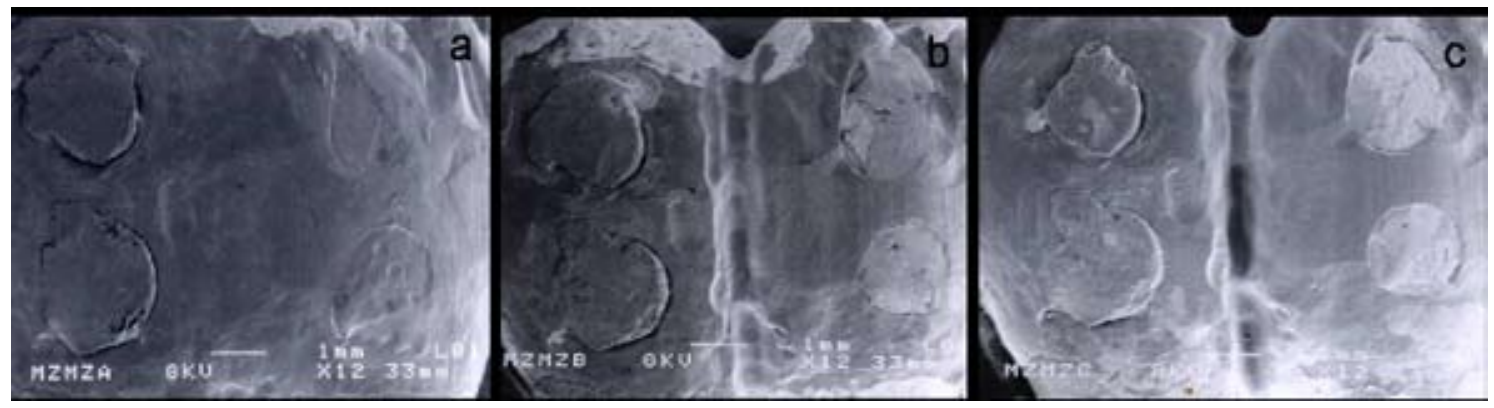

FIGURA 2 - Superfície das réplicas das restaurações antes da aplicação do jato abrasivo (a), após uma aplicação do jato abrasivo (b) e após duas aplicações do jato abrasivo (c). 
Pinto LS, Cordeiro RCL, Lima LM, Marker VA

AVALIAÇÃO DA SUPERFÍCIE DE RESTAURAÇÕES RESINOSAS EXPOSTAS AO ÓXIDO DE ALUMÍNIO

Tabela 2 - Resumo da análise de variância de Friedman aplicada aos dados obtidos na análise das restaurações de resina composta.

\begin{tabular}{l|c|c|c}
\hline \multicolumn{1}{c}{} & $\begin{array}{c}\text { Réplica } \\
1\end{array}$ & $\begin{array}{c}\text { Réplica } \\
2\end{array}$ & $\begin{array}{c}\text { Réplica } \\
3\end{array}$ \\
\hline Soma dos Ranks & 40,00 & 37,00 & 43,00 \\
\hline Mediana & 1,00 & 1,00 & 1,00 \\
\hline Média dos Ranks & 2,00 & 1,85 & 2,15 \\
\hline Média dos valores & $1,27 a$ & $1,25 a$ & $1,40 a$ \\
\hline Desvio padrão & 0,34 & 0,41 & 050 \\
\hline Friedman (Fr) & & 0,90 \\
\hline Graus de liberdade & \multicolumn{3}{|c}{2,00} \\
\hline (p) & \multicolumn{3}{|c}{0.63} \\
\hline
\end{tabular}

p>0,05: não houve diferença estatística significativa.

e criando rugosidades na superfície da restauração a ser reparada ${ }^{2,6,8,17}$. No entanto, ainda não está claro se esta rugosidade produzida pelas partículas abrasivas, denominada condicionamento mecânico, é capaz de alterar a integridade superficial dos selantes e restaurações adjacentes à área a ser reparada. Além disso, é preocupante o fato de que o aumento da rugosidade da superfície favorece a adesão inicial de células e conseqüentemente um maior acúmulo de biofilme ${ }^{22}$.

Em 1981, Katora et al. ${ }^{15}$ analisaram em microscopia eletrônica de varredura, a superfície de dentes que foram abrasonadas por 15 segundos, constatando uma grande irregularidade produzida pelo pó abrasivo. Swift Jr. et al. ${ }^{23}$ (1992), avaliando o efeito da abrasão no reparo de resinas compostas aplicaram o jato abrasivo por 5 e 10 segundos sobre as superfícies a serem reparadas e observaram, também em microscopia eletrônica de varredura, que a abrasão causou substancial rugosidade na superfície das resinas.

Neste estudo, após a análise das restaurações em microscopia eletrônica de varredura, foi possível identificar algumas fraturas em suas margens que foram atribuídas à seqüência de moldagens realizadas, uma vez que estas podem ter forçado a remoção de parte do material restaurador depositado sobre o esmalte (Figura 2). Além disso, na superfície das restaurações foram observados alguns riscos discretos que foram associados ao impacto causado pelo instrumental clínico utilizado para pressionar o material na cavidade.
A influência da rugosidade, produzida pelas partículas de óxido de alumínio, sobre a integridade marginal dos selantes e restaurações adesivas também tem sido questionada. A infiltração marginal verificada em materiais resinosos, aplicados sobre superfícies abrasonadas, parece ser maior que as encontradas nas superfícies condicionadas com ácido ${ }^{11,24}$. Mesmo frente às contradições encontradas entre os trabalhos, o método abrasivo foi considerado em avaliações clínicas tão efetivo quanto o condicionamento ácido na retenção de selantes na superfície oclusal ${ }^{14}$.

Os resultados das pesquisas sobre a efetividade da rugosidade produzida pela abrasão a ar na força de união de materiais resinoso à superfície de esmalte são contraditórios. Valores reduzidos de força de união após o preparo da superfície do esmalte com jatos abrasivos ${ }^{3,7,13}$ e valores semelhantes entre o prétratamento com abrasão ou condicionamento ácido ${ }^{1 \text {, }}$ ${ }^{16}$ foram reportados.

No presente estudo analisou-se o efeito cumulativo da deposição de óxido de alumínio sobre a superfície de restaurações de resina composta e selante resinoso após uma e duas aplicações de jato de óxido de alumínio sobre a superfície dos dentes. Embora alguns espécimes tenham apresentado uma discreta rugosidade superficial após a segunda aplicação do jato de óxido de alumínio, a análise dos resultados evidenciou que não houve diferença estatística significativa entre as superfícies dos materiais analisados, antes e após a utilização do jato de óxido de alumí-

\section{Tabela 3 - Resumo da análise de variância de Friedman aplicada aos dados obtidos na análise das restaurações de selante resinoso.}

\begin{tabular}{l|c|c|c}
\hline & $\begin{array}{c}\text { Réplica } \\
1\end{array}$ & $\begin{array}{c}\text { Réplica } \\
2\end{array}$ & $\begin{array}{c}\text { Réplica } \\
3\end{array}$ \\
\hline Soma dos Ranks & 41,00 & 39,50 & 39,50 \\
\hline Mediana & 1,50 & 1,50 & 1,25 \\
\hline Média dos Ranks & 2,05 & 1,97 & 1,97 \\
\hline Média dos valores & $1,45 a$ & $1,45 a$ & $1,42 a$ \\
\hline Desvio padrão & 0,42 & 0,45 & 0,46 \\
\hline Friedman (Fr) & & 0,07 \\
\hline Graus de liberdade & \multicolumn{3}{|c}{2,00} \\
\hline (p) & \multicolumn{3}{|c}{0,96} \\
\hline
\end{tabular}

p>0,05: não houve diferença estatística significativa. 
nio. Esta observação provavelmente se deve ao fato de o jato não ter sido aplicado diretamente sobre os materiais restauradores, tendo estes sido atingidos apenas pelas partículas periféricas que devido a sua baixa energia cinética não foram capazes de produzir alterações visíveis em microscopia eletrônica de varredura.

Evidências demonstram que a efetividade de corte do jato abrasivo diminui à medida que o spray de afasta da ponta ${ }^{21}$. O jato tem a forma de um triângulo com o ápice voltado para a ponta ativa do aparelho. Quanto mais distante a ponta ativa é colocada do dente, maior a largura da base e conseqüentemente maior a área do dente atingida. Desta forma, as partículas de óxido de alumínio localizadas na periferia do jato têm menor eficiência de corte devido a menor velocidade de propagação e atingem a superfície a ser cortada com menor energia cinética resultando em uma grande deposição de pó no campo operatório ${ }^{18}$.

\section{Conclusão}

As partículas de óxido de alumínio que se depositaram sobre as restaurações durante a utilização do sistema de abrasão a ar não produziram alterações nas superfícies dos materiais restauradores.

\begin{abstract}
The aim of this in vitro study is to evaluate the effect of oxide aluminum oxide powder deposition on restorations adjacent to the area abraded by air abrasion system. It was used 10 third molars that were sectioned and included in wax leaving the buccal surface exposed. In each specimen 4 cavities of approximately $2 \mathrm{~mm}$ of diameter were prepared halfway from a central line demarcated in the wax. The 40 cavities were randomly restored with composite resin (Silux-3M) and resinous sealant (Fluoroshield- Dentsply). Soon afterwards, replica of each specimen was obtained through impression with silicon (Imprint II-3M) and model with epoxy resin (Stycast 1266- Emerson \& Cummings). In the sequence, 2 applications of the abrasive jet were made in the central part of the specimens using PrepStar ${ }^{\circledR}$ regulated to 80 psi, tip with $45^{\circ}$ angulations and $0.48 \mathrm{~mm}$ of internal diameter positioned to $5 \mathrm{~mm}$ of the surface. At the end of each application, the surfaces were washed and dried to get epoxy resin model. All of the replicas were analyzed in SEM by 2 calibrated examiners that were blind for the restoration type. Data, tabulated in scores, were statistically analyzed by Friedman's variance analysis. The results showed no differences statistically significant between the restoration surfaces before and after the use of aluminum oxide powder $(\mathrm{p}>0.05)$. Once the aluminum oxide powder was not applied directly to the restorations, probably the low kinetic energy of the peripheral particles was not capable to produce alterations on material surfaces.
\end{abstract}

\title{
UNITERMS
}

Air Abrasion, dental; dental restoration wear; aluminum oxide.

\section{REFERÊNCIAS}

1. Berry III EA, Ward M. Bond strength of resin composite to air-abraded enamel. Quintessence Int 1995 Aug.; 26(8):559-62.

2. Bouschlicher MR, Reinhardt JW, Vargas MA. Surface treatment techniques for resin composite repair. Am J Dent 1997 Dec.; 10(6):279-83.

3. Browm JR, Barkmeier WW. A comparison of six enamel treatment procedures for sealant bonding. Pediatr Dent 1996 Jan.-Feb.; 18(1):29-31.

4. Chan DC, Summitt JB, Garcia-Goddoy F, Hilton TJ, Chung KH. Evaluation of different methods for cleaning and preparing occlusal fissures. Oper Dent 1999 Nov.-Dec.; 24(6):331-6.

5. Christensen GJ. Air abrasion tooth cutting: State of the art 1998. J Am Dent Assoc 1998 Apr.; 129(4):484-5.

6. Christensen GJ. Cavity preparation: cutting or abrasion? J Am Dent Assoc 1996 Nov.; 127(11):1651-4.

7. Ellis RW, Latta MA, Westerman GH. Effect of air abrasion and acid etching on sealant retention: an in vitro study. Pediatr Dent 1999 Sep.Oct.; 21(6):316-9.
8. Feinman RA. High velocity air microabrasion for conservative tooth preparation: the principle and the clinical procedure. Pract Period Aesthet Dent 1995 Oct.; 7(8):37-42.

9. Goldstein RE, Parkins FM. Air-abrasive technology: its new role in restorative dentistry. J Am Dent Assoc 1994 May; 125(5):551-7.

10. Goldstein RE, Parkins FM. Using air-abrasive technology to diagnose and restore pit and fissure caries. J Am Dent Assoc 1995 Jun.; 126(6):761-6.

11. Guirguis R, Lee J, Conry J. Microleakage evaluation of restorations prepared with air abrasion. Pediatr Dent 1999 Sep.-Oct.; 21(6):311-5.

12. Haws SM, Oliveira ML, Vargas MA, Kanellis MJ. Air-abrasion and microleakage of pit and fissure selants [abstract n.1301]. J Dent Res 1996; 75(sp.Issue): 180.

13. Hogervorst WL, Feilzer AJ, Prahl-Andersen B. The air-abrasion technique versus the conventional acid-etching technique: A quantification of surface enamel loss and a comparation of shear bond strength. Am J Orthod Dentofac Orthop 2000 Jan.; 117(1):20-6. 
14. Kanellis MJ, Warren JJ, Levy MS. Comparison of air abrasion versus acid etch sealant techniques: six-month retention. Pediatr Dent 1997 May-Jun.; 19(4):258-61.

15. Katora ME, Jubach T, Polimus MM. Airbrasive etching of the enamel surface. Quintessence Int 1981 Sep.; 12(9):967-8.

16. Keen DS, Von Fraunhofer FM, Parkins FM. Air-abrasive "etching": composite bond strengths [abstract n.238]. J Dent Res 1994; 73(sp. Issue): 131

17. Kupiec KA, Barkmeier WW. Laboratory evaluation of treatments for composite repair. Oper Dent 1996 Mar.-Apr.; 21(2):59-62.

18. Laurell KA, Hess JA. Scanning electron micrographic effects of air abrasion cavity preparation on human enamel and dentin. Quintessence Int 1995 Feb.; 26(2):139-44.

19. Natoli S. Air abrasion in dentistry. Dent Assist 1997 Sep.-Oct.; 66(5):11-3.

20. Roeder LB, Berry III EA, You C, Powers JM. Bond strength of composite to air-abraded enamel and dentin. Oper Dent 1995 Sep.-Oct.; 20(5):186-90.

21. Santos-Pinto L, Peruchi C, Marker VA, Cordeiro R. Effect of handipiece tip design on the cutting efficiency of an air abrasion system. Am J Dent 2001 Dec.; 14(6):397-401, 2001.
22. Shintani H, Satou J, Satou N, Hayashihara H, Inoue T. Effects of various finishing methods on staining and accumulation of Streptococcus mutans HS-6 on composite resin. Dent Mat 1985 Dec.; $1(6): 225-7$

23. Swift Jr EJ, Levalley BD, Boyer DB. Evaluation of new methods for composite repair. Dent Mater 1992 Nov.; 8(6):362-5.

24. Zyskind D, Zyskind K, Hirschfeld Z, Fuks AB. Effect of etching on leakage of sealants placed after air abrasion. Pediatr Dent 1998 Jan.Feb.; 20(1):25-7.

Recebido em 08/12/06 Aprovado em 10/10/07

Correspondência: Profa. Dra. Lourdes dos Santos-Pinto Av. Queiroz Filho, 972 - Bairro Vila Harmonia 14802-610 - Araraquara - SP - Brasil e-mail: 1spinto@foar.unesp.br 